\title{
Early-life intestinal microbiome in Trachemys scripta elegans analyzed using 16S rRNA sequencing
}

\author{
Qin Peng ${ }^{1}$, Yahui Chen ${ }^{1}$, Li Ding $^{1}$, Zimiao Zhao ${ }^{1}$, Peiyu Yan ${ }^{1}$, Kenneth B. Storey ${ }^{2}$, Haitao Shi $^{1}$, Meiling Hong \\ Corresp. 1 \\ ${ }^{1}$ Ministry of Education Key Laboratory for Ecology of Tropical Islands, College of Life Sciences, Hainan Normal University, Haikou, Hainan, China \\ 2 Department of Biology, Carleton University, Ottawa, Canada \\ Corresponding Author: Meiling Hong \\ Email address: mlhong@hainnu.edu.cn
}

During the early-life period, the hatchlings of red-eared slider turtles (Trachemys scripta elegans) rely on their own post-hatching internal yolk for several days before beginning to feed. The gut microbiome is critical for the adaptation of organisms to new environments, but, to date, how the microbiome taxa are assembled during early life of the turtle is unknown. In this study, the intestinal microbiome of red-eared slider hatchlings (fed on commercial particle food) was systematically analyzed at four different growth stages ( $0 \mathrm{~d}$, 10d, 20d, 30d) by a high-throughput sequencing approach. Results showed that the dominant phyla were Firmicutes (58.23\%) and Proteobacteria $(41.42 \%)$ at 0-day, Firmicutes (92.94\%) at 10-day, Firmicutes (67.08\%) and Bacteroidetes (27.17\%) at 20-day, and Firmicutes (56.46\%), Bacteroidetes (22.55\%) and Proteobacteria (20.66\%) at 30-day post-hatching. Members of the Bacteroidaceae family were absent in 0-day and 10-day turtles, but dominated in 20-day and 30-day turtles. The abundance of Clostridium also showed the highest value in 10-day turtles. The richness of the intestinal microbiomes was lower at 0-day and 30-day than that at 10-day and 20-day, while the diversity was higher at 10-day and 30-day than that at 0-day and 20-day. The results endowed the turtles with an ability to enhance their tolerance to the environment. 
1 Early-life intestinal microbiome in Trachemys scripta elegans analyzed using 16S rRNA 2 Sequencing

3

4 Qin Peng ${ }^{1}$, Yahui Chen ${ }^{1}$, Li Ding ${ }^{1}$, Zimiao Zhao ${ }^{1}$, Peiyu Yan ${ }^{1}$, Kenneth B. Storey ${ }^{2}$, Haitao Shi ${ }^{1}$, 5 Meiling Hong ${ }^{1 *}$

6

$7 \quad{ }^{1}$ Ministry of Education Key Laboratory for Ecology of Tropical Islands, College of Life

8 Sciences, Hainan Normal University, Haikou, China

$9{ }^{2}$ Department of Biology, Carleton University, Ottawa, Canada

10

11

12

13

14

15

16

17

18

19

20

21

22

23

24

25

26

27

28

29

30

31

32

33

34

35

36

37

*Corresponding Author:

Meiling Hong

South Longkun Road, Haikou, Hainan, 571158, China

Email address: mlhong@hainnu.edu.cn 
38

39

40

41

42

43

44

45

46

47

48

49

50

51

52

53

54

55

56

57

58

59

60

61

\section{Abstract}

During the early-life period, the hatchlings of red-eared slider turtles (Trachemys scripta elegans) rely on their own post-hatching internal yolk for several days before beginning to feed. The gut microbiome is critical for the adaptation of organisms to new environments, but, to date, how the microbiome taxa are assembled during early life of the turtle is unknown. In this study, the intestinal microbiome of red-eared slider hatchlings (fed on commercial particle food) was systematically analyzed at four different growth stages $(0 \mathrm{~d}, 10 \mathrm{~d}, 20 \mathrm{~d}, 30 \mathrm{~d})$ by a high-throughput sequencing approach. Results showed that the dominant phyla were Firmicutes (58.23\%) and Proteobacteria $(41.42 \%)$ at 0 -day, Firmicutes $(92.94 \%)$ at 10-day, Firmicutes $(67.08 \%)$ and Bacteroidetes (27.17\%) at 20-day, and Firmicutes (56.46\%), Bacteroidetes (22.55\%) and Proteobacteria $(20.66 \%)$ at 30 -day post-hatching. Members of the Bacteroidaceae family were absent in 0-day and 10-day turtles, but dominated in 20-day and 30-day turtles. The abundance of Clostridium also showed the highest value in 10-day turtles. The richness of the intestinal microbiomes was lower at 0-day and 30-day than that at 10-day and 20-day, while the diversity was higher at 10-day and 30-day than that at 0-day and 20-day. The results endowed the turtles with an ability to enhance their tolerance to the environment.

\section{Subjects: Microbiology; Zoology}

Key words: Red-eared slider; gut microbiota; microbiome diversity; microbiome composition 


\section{INTRODUCTION}

The microbial community in the gastrointestinal tract has a major role to play in several physiology processes of the host, such as maintaining intestinal microecological balance, promoting host health, and providing nutrients (Chung et al. 2012; Kahrstrom et al. 2016; Mohd Shaufi et al. 2015; Yamashiro 2017). In addition, the gut microbiome is also associated with gut disease, feed conversion, parasite colonization, and immune system activity of the host (Li et al. 2017; Singh et al. 2012). Gut microbes can prevent the overgrowth of gut pathogens by building a natural barrier, called 'colonization resistance' that can inhibit the growth of pathogenic bacteria by occupying the same ecological niche (Buffie \& Pamer 2013; Ducarmon et al. 2019; Scott et al. 2015). Early promotion of nutrient metabolism and innate immune response depend upon the bacterial species that colonize the digestive tract. Therefore, research on the microbiota of animals is gaining popularity in order to understand the relationships between host health, immunity, and disease resistance.

The red-eared slider turtle (Trachemys scripta elegans), with a native range in the southeastern U.S.A. and northeastern Mexico, is now one of the most successful invasive species in many regions of the world (Mali et al. 2015). It has been reported to outcompete several native freshwater turtles in Europe and Asia, including Mauremys sinensis, Mauremys leprosa, and Mauremys reevesii (Nishizawa et al. 2014; Polo-Cavia et al. 2012). Several studies have been conducted in an effort to understand the invasion mechanism of the red-eared slider, including examining their feeding kinematics (Nishizawa et al. 2014), home range (Ma et al. 2013), food snatch ability, and hunger endurance ability (Zhao et al. 2013). Compared to native species, redeared sliders exhibit unique advantages including holding an ability to live in brackish water that allows them to disperse along coastlines (Yang \& Shi 2014), greater food-competing ability, higher tolerance of starvation, higher reproductive capacity, and a greater ability to adapt to different environment by exploit new food sources or supplies (Ma et al. 2013; Zhao et al. 2013). Several of these advantages might be associated with the gut microbiome, that can influence food digestion and nutrient absorption, but little is known about the adaptive capacity of the intestinal microbiome of red-eared sliders. The existing studies on this species are limited to culture-dependent phenotypic and biochemical characterization of the gut microorganisms, and are not sufficient for systematically understanding the gut microecosystem of red-eared sliders (Gioia-Di Chiacchio et al. 2014; James et al. 2008).

High-throughput analysis of bacteria diversity and abundance can provide a greater understanding of the gut microecosystem of an animal host and potentially contribute to understanding the invasive potential of different species (Ahasan et al. 2017; Han et al. 2015; Kumar et al. 2015; McLaughlin et al. 2015; Qin et al. 2010; Sergeant et al. 2014; Zeng et al. 2015). The community of the gut microbiome varies at different growth stages (Arizza et al. 2019; Burgos 
et al. 2018; Campos et al. 2018; Dulski et al. 2018; Huang et al. 2014). The onset of feeding is a critical stage in the development of animals (Sarasquete et al. 1995). During the early-life period, turtles rely on their own post-hatching yolks (absorbed into the coelomic cavity) for several days, and then begin to feed. By selecting for bacteria associated with this change of nutrition access, the host is thought to derive a benefit in the form of increased absorption efficiency (Rawls et al. 2004). Although there have been some studies on the gastrointestinal microbiome of sea turtles (Ahasan et al. 2017; Price et al. 2017), as far as is known, there is a lack of studies relation to gut microbiome of Trachemys scripta elegans. Therefore, the present study analyzes, for the first time, the changes in the intestinal bacterial community of red-eared sliders during the post-hatching period via the use of a high-throughput sequencing approach. Our results provide insights into the changes in intestinal bacterial communities in the early life of this invasive turtle species, and provide basic knowledge on host-bacteria associations in turtles.

\section{MATERIALS AND METHODS}

\section{Animals and sample collection}

Twelve red-eared sliders were randomly selected from the Hongwang turtle farm (Hainan, China) after they were hatched immediately. The newly hatched turtle was still placed in incubator with vermiculite, until its exogenous yolk sac was assimilated almost at day 8-9 after hatched. Then, these remaining nine turtles of 10-day were mixed farmed in a cement pool with exposed tap water and fed with standard diet (turtle food, Inch-Gold, China). At $0 \mathrm{~d}, 10 \mathrm{~d}, 20 \mathrm{~d}, 30 \mathrm{~d}$ after hatching, these turtles were measured by vernier caliper for their body heights, carapace lengths and carapace widths, and weighed for their body weights. Then three turtles were randomly selected and anesthetized at $-20^{\circ} \mathrm{C}$ cryoanesthesia for 30-60 minutes. All turtles were euthanized by decapitation, and the intestinal tracts were collected separately and frozen immediately in liquid nitrogen. Water samples were also collected from random sites in the pool in which the turtles of 10-day, 20-day, and 30-day were living. All samples were stored at $-80^{\circ} \mathrm{C}$ and used for extraction of total DNA.

\section{DNA extraction and 16S rRNA gene sequencing}

Total genomic DNA was extracted from intestine samples and environmental water samples using a Stool DNA Kit (OMEGA Bio-Tek, Norcross, GA) as per the manufacturer's instructions. Then V4-V5 region of bacterial 16S rRNA gene were amplified by using barcode primers $515 \mathrm{~F}$ (5'-GTGCCAGCMGCCGCGGTAA-3') and 907R (5'-CCGTCAATTCMTTTRAGTTT-3') (Sun et al. 2013; Yu et al. 2019). The amplicons were pooled, purified and then quantified using a Nanodrop (Thermo Scientific, MA). The purified amplicons were used for next-generation sequencing using Illumina Hiseq2500 PE250 by Sagene Biotech Inc. (Guangzhou, China).

\section{Data analysis}


134

135

136

137

138

139

140

141

142

143

144

145

146

147

148

149

150

151

152

153

154

155

156

157

158

159

160

161

162

163

164

165

166

167

168

169

The reads archived by high-throughput sequencing were filtered to remove both those containing ambiguous nucleotides and primers sequences. The clean reads were used for further data analysis. Sequence sets showing 97\% identities were defined as an Operational Taxonomic Unit (OTU) and were used for diversity (Shannon index and Simpson index) (Simpson 1949), and richness (Ace index and Chaol index) (Chao 1984) analysis using QIIME (Caporaso et al. 2010). Rarefaction curves, alpha diversity, and beta diversity calculations were also analyzed using QIIME (Caporaso et al. 2010). Taxonomic assignments of OTUs that reached the $97 \%$ similarity level were made using the QIIME software package through comparison with SILVA (Quast et al. 2013), Greengene (DeSantis et al. 2006) and Ribosomal Database Project (RDP) databases (Wang et al. 2007). A heat map was generated using the heat map function in R (http://www.r-project.org/) with row normalized. The similarity among the microbial communities was determined using weighted UniFrac distances analysis in principal coordinate analysis ( $\mathrm{PCoA}$ ) was performed (Lozupone \& Knight 2005). The linear discriminant analysis (LDA) effect size (LEfSe) method was used to identify the most differentially abundant taxons between groups, which would help discover biomarkers (Segata et al. 2011). Taxa were regarded as being a statistically different biomarker when the LDA scores were $\geq 4$. Statistical analysis was performed using ANOVA for morphological index, and Linear General Model for the diversity and richness index with initial body weight as the covariate. The value of $P<0.05$ was considered to be statistically significant.

\section{Nucleotide sequence accession numbers.}

Raw sequences for this project were deposited in NCBI non-redundant nucleotide database under the SRA accession number

SRP154277 (https://doi.org/10.6084/m9.figshare.9204992.v1).

\section{Ethical Statement}

Experimental animal procedures had the prior approval of the Animal Research Ethics Committee of Hainan Provincial Education Centre for Ecology and Environment, Hainan Normal University (permit no. HNECEE-2014-004, as defined by Chinese regulations).

\section{RESULTS}

Turtle growth

For the first 10 days after hatching, turtles were raised without feeding, because of the existence of post-hatching yolk. During this period, the turtles showed a slight decrease in their body height. However, body weight, carapace length and width showed no significant change over this time ( $p>0.05$ ). However, after growing for 20 or 30 days, body weight, carapace length and width were significantly increased $(p<0.05)$ (Table 1$)$. There were significant differences in body height between 0 -day Vs 10-day, 10-day Vs 30-day, and 20-day Vs 30-day $(p<0.05)$.

\section{The diversity and richness index}


Among the 12 samples of gastrointestinal tract, a total of 578,559 qualified reads, with an average of 48,213 reads per sample were archived (Table 2). Analysis of the qualified reads lead to the classification of 15,173 OTUs, with an average of 1264 OTUs per sample. Both Shannon index and Simpson index showed the within-habitat diversity of samples. The Shannon index is most sensitive to changes in the importance of the rare species in the sample, while the Simpson index is most sensitive to changes in the most abundant species in the sample. Both Shannon index and Simpson index were higher at 10-day, 30-day, and water than that at 0-day and 20-day (Fig. $\mathrm{S} 1 \mathrm{~A}$ and S1B). Based on the analysis of Ace and Chao1, which represent the richness of OTU in a bacterial community, and both were higher at 10-day and 20-day than that at 0-day, 30-day, and water (Fig. S1C and S1D).

To better visualize the relationships among samples, an MDS plot was generated with all replicates and sampling points clustered by development stage (Fig.1). Based on the ordination of the weighted Unifrac distances, different gut microbiome distribution among these samples from different time. A significant difference was found between the microbiome of the water in the holding tank and turtle intestines. Moreover, microbiomes at 10-day post-hatching formed a tight cluster that did not overlap with other samples. The turtles at 0-day also displayed a significantly different microbial community although closer to those exhibited by the turtles at 10 -day. The microbial communities overlapped between 20-day and 30-day but were distinct from the 0-day and 10-day.

\section{Abundance and significant differences between the four growth stages at the phylum level}

Among these samples, the top 15 phyla were represented in Fig. 2A, and the most prominent phyla were listed in Fig. 2B and Table S1. Among the newly hatched turtles, the dominant phylum was Firmicutes (58.23\%), followed by Proteobacteria (41.42\%), and then Bacteroidetes (0.32\%). At 10-day, the percentage of Firmicutes increased greatly to $92.94 \%$ whereas Proteobacteria decreased sharply to $5.68 \%$, and no significant change was seen for Bacteroidetes $(0.31 \%)$. At 20 day, Bacteroidetes increased strongly (27.17\%) while Firmicutes (67.08\%) decreased, and Proteobacteria remained relative stable (5.74\%). At 30-day, the dominant phyla were Firmicutes (56.46\%), Proteobacteria (20.66\%), and Bacteroidetes (22.55\%). Furthermore, the ratio of Firmicutes to Bacteroidetes 0-day, 10-day, 20-day, and 30-day was 181.97, 299.80, 2.47, and 2.50, respectively. In samples of environmental water, Proteobacteria (70.33\%), Bacteroidetes (23.24\%), Actinobacteria (4.45\%), and Firmicutes (1.90\%) were found to be the major bacterial phyla represented.

\section{Abundance and significant differences between the four growth stages at the family level}

A total of 166 bacterial families were identified and the most abundant families are shown in Fig. 3A and Table S2. At 0-day, the dominant families were Clostridiaceae (13.03\%), Enterobacteriaceae (23.52\%), and Paenibacillaceae (36.01\%). Along with the growth of the turtles, 
206

207

208

209

210

211

212

213

214

215

216

217

218

219

220

221

222

223

224

225

226

227

228

229

230

231

232

233

234

235

236

237

238

239

240

241

the bacteria from Paenibacillaceae sharply decreased at 10-day $(0.86 \%)$ and disappeared at 20-day and 30-day. At 10-day, bacteria belonging to the families Lachnospiraceae (33.75\%), Clostridiaceae (30.24\%), Peptostreptococcaceae (19.94\%), Staphylococcaceae (4.07\%), Moraxellaceae (3.75\%) were the dominant intestinal components. Except for the Moraxellaceae family, all the other families belong to the Firmicutes phylum and made up to $92.94 \%$ of the total intestinal microbiome. At 20-day, the dominant families were Lachnospiraceae (27.74\%), Bacteroidaceae (27.36\%), Peptostreptococcaceae (23.43\%), Clostridiaceae (9.39\%), Enterobacteriaceae (5.34\%), and Ruminococcaceae (3.00\%). Although the Clostridiaceae increased sharply during the first 10 days, it had decreased significantly at 20-day. By contrast, Bacteroidaceae family bacteria, which were absent in 10-day turtles were a dominant group in intestine of 20-day and 30-day old turtles. At 30-day, the dominant families were Bacteroidaceae (22.56\%), Clostridiaceae (18.13\%), Peptostreptococcaceae (17.60\%), Enterobacteriaceae (12.56\%), and Lachnospiraceae (8.18\%). In brief, the families Lachnospiraceae, Ruminococcaceae and Peptostreptococcaceae were also major bacterial components in intestines of turtles older than 10 days. The water microbiome showed the obviously different with the gut microbiome (Fig. 3B; Table S2). Comamonadaceae (22.86\%), Aeromonadaceae (12.18\%), Weeksellaceae (8.95\%), Xanthomonadaceae (7.84\%), Cytophagaceae $(7.71 \%)$ were the dominant families in the water microbiome, while the relative of abundance of these families bacteria was very low.

\section{Abundance and significant differences between the four growth stages at the genus level}

At the genus level, the detected sequences from all samples were assigned into 323 genera. The genus Clostridium dominated in all the tested turtle samples, ranging from $8.23 \%$ to $24.98 \%$ (Table S3). At 0-day, the most abundant genera were Paenibacillus (36.01\%), Citrobacter (20.78\%), Clostridium (11.95\%), and Achromobacter (10.75\%). At 10-day, Clostridium (24.98\%) and Epulopiscium (31.91\%) were the dominant genera. The relative abundance of Epulopiscium in 10-day turtles was $31.91 \%$, higher than that in 0-day (0.15\%), 20-day (1.42\%) and 30-day $(0.72 \%)$. Similarly, the relative abundance of Clostridium was also highest in 10-day, and decreased to $8.23 \%$ at 20 -day and $17.5 \%$ at 30 -day.

\section{Specific bacterial communities at each growth stage}

The specific OTU numbers of 0-day, 10-day, 20-day, and 30-day turtles were 1299, 2845, 2509 , and 1704, respectively. Among these identified OTUs, only 47 of them were shared by all turtles (Fig. 4; Table S4). These core OTUs belonged to three families, the Lachnospiraceae, Peptostreptococcaceae, and Clostridiaceae with relative abundances of $9 \%, 23 \%$, and $68 \%$, respectively. There were only 148 conserved OTUs between the intestines of 0-day and 30-day turtles, whereas 630 OTUs were conserved between the 20-day and 30-day.

To identify the specific bacteria in the intestines of turtles at different stages, biomarkers of 
242 bacteria belonging to different taxonomic groups were analyzed (Fig. S2; Fig. 5; Table S5). At

243 family level, the significant different families were Paenibacillaceae and Alcaligenaceae at 0-day,

244 Lachnospiraceae at 10-day, Bacteroidaceae and Odoribacteraceae at 20-day, and

245 Erysipelotrichaceae at 30-day. At genus level, Paenibacillus and Achromobacter (0-day),

246 Epulopiscium (10-day), Blautia (30-day) showed the significant difference in the 0-day, 10-day

247 and 30-day turtles, respectively, whereas no genus reached a taxonomic level score (LDA score $\geq$

248 4) in 20-day turtles. In summary, these different taxa could be used as distinguishing biomarkers.

249 The healthy red-eared slider revealed 10 such microbial biomarkers that showed significant

250 differences in abundance at different growth stages in the standardized condition without

251 considering other factors.

252

253

\section{DISCUSSION}

254

255

In this study, the first detailed analysis of the intestinal bacterial communities of the red-eared slider turtle over the first 30 days post-hatching was performed using a high-throughput sequencing

256

257

258

259

260

261

262

263

264

265

266

267

268

269

270

271

272

273

274

275

276

277 approach. During the first 9 days post-hatch, the turtles were fasting and acquired nutrition from the yolk sac and, hence, did not need to digest and assimilate nutrition via the intestine. Therefore, the richness of the intestinal microbiome was the lowest at 0-day and growth before the first feeding was very slow. After the closure of their umbilical fontanel, the turtles were fed with a commercial diet. In our study, the 10-day turtles showed the highest intestinal microbiome diversity and abundance. Very short dietary manipulations can have a rapid and substantial impact on the intestinal microbiome (David et al. 2014). This was consistent with other researches that diet could affect intestinal microbial diversity (Claesson et al. 2012; Scott et al. 2013). The results suggested that the microbiome of the turtle adjusts itself to optimize the bacterial species needed for the particular food that is given which is in this case is a constant single food. Another explanation was the richness and diversity of gut microbes varied with development stage in turtles. This is consistent with previous reports showing that the diversity and richness of gut microbiome of pigs and chicks decreased with age (Ballou et al. 2016; Zhao et al. 2015). For the 30-days turtles, the intestinal microbiomes diversity increased again (Table 2), which endowed the turtles with ability to face to different diet type and enhanced their tolerance to the environment.

The results revealed that the gut microbiome of the turtle exhibited temporal differences in composition at the genus level and beyond. In the intestinal microbiome of 10-day turtles, bacteria from the Epulopiscium genus showed the highest relative abundance. Epulopiscium is a group of giant bacteria that was reported to be widely distributed in the intestinal tracts of herbivorous surgeonfish with high abundance (Miyake et al. 2016). The predicted function of Epulopiscium is to facilitate the digestion and decomposition of ingested food (Flint et al. 2008; Thomas et al. 2011). These results suggested that Epulopiscium bacteria might facilitate the turtle to access 
278

279

280

281

282

283

284

285

286

287

288

289

290

291

292

293

294

295

296

297

298

299

300

301

302

303

304

305

306

307

308

309

310

311

312

313

nutrition from the diet. After the fasting time (during yolk consumption), the turtles were fed with commercial food, which lead to changes in the intestinal microbiome composition and formed intestinal microbiota that were suitable for digesting the commercial diet.

At family level, the Bacteroidaceae, Lachnospiraceae, and Peptostreptococcaceae were the dominant bacteria in the turtles after growing for 10 days. Bacteria of these families are responsible for metabolizing carbohydrate complexes, such as cellulose (Price et al. 2017), and degrading plant material to maintain gut health in several animals (Amy Biddle 2013). These dominant families were perhaps a good preparation by the gut for the foods that the juvenile turtles will begin to ingest, which was fitting well with the starvation tolerance behavior of this kind of turtle.

Many studies have shown that Bacteroidetes, Firmicutes and Proteobacteria are numerically dominant phyla in the gut microbiome of animals (Thomas et al. 2011), including humans (Qin et al. 2010), wild-captured green turtles (Chelonia mydas) (Ahasan et al. 2017), loggerhead turtles (Caretta caretta) (Abdelrhman et al. 2016), doves, waterfowls and geese (Hird et al. 2015; Li et al. 2017) and other vertebrates (Ley et al. 2008). In the present study, although Proteobacteria made up a large proportion of the sequences in the intestinal microbiome of 0-day turtles, overall, the Firmicutes and Bacteroidetes were the most ubiquitous and common, which is consistent with previous reports. The relative abundance of Bacteroidetes phylum was extremely low during the first 10 days, and then rapidly increased to a dominant phylum at 20-day and 30-day. Bacteroidetes is a degrader of polymeric organic matter to help the host digest food. The interaction between Bacteroidetes and the host is known to be mutualism since the fitness of both partners is increased (Backhed et al. 2005). Bacteroidetes also function to activate T-cell mediated responses (Mazmanian 2008), limit the colonization of the potential pathogens (Mazmanian et al. 2008), and produce antineoplastic properties (Thomas et al. 2011). Bacteroidetes can colonize all parts of gastrointestinal tract, despite of the differences in ambient $\mathrm{pH}$, nutrients, and oxygen availability in different parts (Thomas et al. 2011). So, the increase of Bacteroidetes with the turtles grows may endow the host with stronger ability to digest food and resist disease. Species of the Firmicutes phylum can process food with a higher abundance of insoluble carbohydrate (Jandhyala et al. 2015). A recent study showed that the ratio of Firmicutes to Bacteroidetes was of significant relevance in signaling human gut microbiota status (Mariat et al. 2009), the relative proportion of Bacteroidetes decreasing in unhealthy humans (Ley et al. 2006). Another research revealed higher relative abundance of Bacteroidetes could help keep the turtle healthy (Ahasan et al. 2018). In our study, the ratio of Firmicutes to Bacteroidetes at 0-day and 10-day was much higher than at 20day and 30-day, which indicated that it tended to be stable in the older turtles, and the relative proportion of Bacteroidetes increased. So, a stable composition of Firmicutes and Bacteroidetes and a relatively higher proportion of Bacteroidetes could maintain intestinal stability to make the 
314

315

316

317

318

319

320

321

322

323

324

325

326

327

328

329

330

331

332

333

334

335

336

337

338

339

340

341

342

343

344

345

346

347

348

349

turtle healthier, and allow it to adapt to environmental surroundings and food availability more quickly. Several factors, including host intestinal structure, physiological status, habitat, developmental stages, feeding strategy, living environment, and certain environment conditions, were reported to affect the composition of the host intestinal microbiome (Ahasan et al. 2017; Ravussin et al. 2012). Nevertheless, in the present study, there were no relationship between the water microbiome and the dominant phyla Proteobacteria and Firmicutes of the turtle intestine. Thus, the intestinal bacteria of these phyla were rarely affected by living water environment without considering other factors. So, it can be inferred that the intestinal microbiomes of redeared slider may be self-regulating, which may endow the turtles with the ability to readily adapt into different environments, different foods and seasonal changes. The Proteobacteria phylum was rich in the water environment, but in the intestinal microenvironment, the relative abundance of Proteobacteria was low in 10-day and 20-day turtles, but increased in the 30-day turtle gut. A previous study reported that after Bacteroidetes break down proteins into amino acids, and chitin into N-acetylglucosamine, Proteobacteria could take up and process in these monomers (Cottrell \& Kirchman 2000). Thus, Proteobacteria could help the turtle to get nutrition from degraded monomers. Overall, the dominant existence of Proteobacteria, Firmicutes and Bacteroidetes could be one reason that turtles are well-adapted to a flexible environment. In the wild, food types would vary on a daily or seasonal basis and also with the specific environment, such as lake, river, brackish coastal water, etc. In our study, the living water environment does not affect the gut microbiome of red-eared slider under the standard condition. Based on the literature available in humans and other animal species (Chen et al. 2014; Zha et al. 2018), it is possible that multiple exogenous factor, including food or feed, influences the intestinal microbiota community.

In the family and below level, total 10 biomarkers were discovered in the 0-day, 10-day, 20day and 30-day samples. The biomarkers were obviously different for the different age groups, so it inferred that gut biomarker might help to predict age of this turtle.

\section{CONCLUSIONS}

The diversity and richness of the intestinal microbiomes were the lowest at 0 -day, and highest at 10-day because of its first offer with food. With the turtles growing, the high abundance of Firmicutes, Bacteroidetes, Proteobacteria and the stable ratio of Firmicutes to Bacteroidetes could help maintain intestinal stability to make the red-eared slider healthier, and endow it with high adaptive capacity for dealing with different environments. Peptostreptococcaceae, Lachnospiraceae and Clostridiaceae were core bacterial communities at all growth stages in the red-eared slider. At the class level, Bacilli, Clostridia, Bacteroidia and Erysipelotrichi could be regarded as distinguishing biomarkers in the intestine of 0-day, 10-day, 20-day and 30-day turtles, respectively. 
350

351

352

353

354

355

356

357

358

359

360

361

362

363

364

365

366

367

368

369

370

371

372

373

374

375

376

377

378

379

380

381

382

383

384

385

386

387

388

389

\section{REFERENCES}

Abdelrhman KF, Bacci G, Mancusi C, Mengoni A, Serena F, and Ugolini A. 2016. A First Insight into the Gut Microbiota of the Sea Turtle Caretta caretta. Front Microbiol 7:1060. 10.3389/fmicb.2016.01060

Ahasan MS, Waltzek TB, Huerlimann R, and Ariel E. 2017. Fecal bacterial communities of wildcaptured and stranded green turtles (Chelonia mydas) on the Great Barrier Reef. FEMS Microbiol Ecol 93. 10.1093/femsec/fix139

Ahasan MS, Waltzek TB, Huerlimann R, and Ariel E. 2018. Comparative analysis of gut bacterial communities of green turtles (Chelonia mydas) pre-hospitalization and post-rehabilitation by high-throughput sequencing of bacterial 16S rRNA gene. Microbiol Res 207:91-99. 10.1016/j.micres.2017.11.010

Amy Biddle LS, Jeffrey Blanchard ,Susan Leschine 2013. Untangling the Genetic Basis of Fibrolytic Specialization by Lachnospiraceae and Ruminococcaceae in Diverse Gut Communities. Diversity 5:627-640.

Arizza V, Vecchioni L, Caracappa S, Sciurba G, Berlinghieri F, Gentile A, Persichetti MF, Arculeo M, and Alduina R. 2019. New insights into the gut microbiome in loggerhead sea turtles Caretta caretta stranded on the Mediterranean coast. PLoS One 14:e0220329. 10.1371/journal.pone.0220329

Backhed F, Ley RE, Sonnenburg JL, Peterson DA, and Gordon JI. 2005. Host-bacterial mutualism in the human intestine. Science 307:1915-1920. 10.1126/science.1104816

Ballou AL, Ali RA, Mendoza MA, Ellis JC, Hassan HM, Croom WJ, and Koci MD. 2016. Development of the Chick Microbiome: How Early Exposure Influences Future Microbial Diversity. Front Vet Sci 3:2. 10.3389/fvets.2016.00002

Buffie CG, and Pamer EG. 2013. Microbiota-mediated colonization resistance against intestinal pathogens. Nat Rev Immunol 13:790-801. 10.1038/nri3535

Burgos FA, Ray CL, and Arias CR. 2018. Bacterial diversity and community structure of the intestinal microbiome of Channel Catfish (Ictalurus punctatus) during ontogenesis. Syst Appl Microbiol 41:494-505. 10.1016/j.syapm.2018.04.006

Campos P, Guivernau M, Prenafeta-Boldu FX, and Cardona L. 2018. Fast acquisition of a polysaccharide fermenting gut microbiome by juvenile green turtles Chelonia mydas after settlement in coastal habitats. Microbiome 6:69. 10.1186/s40168-018-0454-z

Caporaso JG, Kuczynski J, Stombaugh J, Bittinger K, Bushman FD, Costello EK, Fierer N, Pena AG, Goodrich JK, Gordon JI, Huttley GA, Kelley ST, Knights D, Koenig JE, Ley RE, Lozupone CA, McDonald D, Muegge BD, Pirrung M, Reeder J, Sevinsky JR, Turnbaugh PJ, Walters WA, Widmann J, Yatsunenko T, Zaneveld J, and Knight R. 2010. QIIME allows analysis of high-throughput community sequencing data. Nat Methods 7:335-336. 10.1038/nmeth.f.303

Chao A. 1984. Nonparametric estimation of the number of classes in a population. Scandinavian Journal of Statistics 11:265-270.

Chen J, He X, and Huang J. 2014. Diet effects in gut microbiome and obesity. J Food Sci 79:R442- 
390

391

392

393

394

395

396

397

398

399

400

401

402

403

404

405

406

407

408

409

410

411

412

413

414

415

416

417

418

419

420

421

422

423

424

425

426

427

428

429

430

451. 10.1111/1750-3841.12397

Chung H, Pamp SJ, Hill JA, Surana NK, Edelman SM, Troy EB, Reading NC, Villablanca EJ, Wang S, Mora JR, Umesaki Y, Mathis D, Benoist C, Relman DA, and Kasper DL. 2012. Gut immune maturation depends on colonization with a host-specific microbiota. Cell 149:1578-1593. 10.1016/j.cell.2012.04.037

Claesson MJ, Jeffery IB, Conde S, Power SE, O'Connor EM, Cusack S, Harris HM, Coakley M, Lakshminarayanan B, O'Sullivan O, Fitzgerald GF, Deane J, O'Connor M, Harnedy N, O'Connor K, O'Mahony D, van Sinderen D, Wallace M, Brennan L, Stanton C, Marchesi JR, Fitzgerald AP, Shanahan F, Hill C, Ross RP, and O'Toole PW. 2012. Gut microbiota composition correlates with diet and health in the elderly. Nature 488:178-184. 10.1038/nature11319

Cottrell MT, and Kirchman DL. 2000. Community composition of marine bacterioplankton determined by $16 \mathrm{~S}$ rRNA gene clone libraries and fluorescence in situ hybridization. Appl Environ Microbiol 66:5116-5122. 10.1128/aem.66.12.5116-5122.2000

David LA, Maurice CF, Carmody RN, Gootenberg DB, Button JE, Wolfe BE, Ling AV, Devlin AS, Varma Y, Fischbach MA, Biddinger SB, Dutton RJ, and Turnbaugh PJ. 2014. Diet rapidly and reproducibly alters the human gut microbiome. Nature 505:559-563. 10.1038 /nature 12820

DeSantis TZ, Hugenholtz P, Larsen N, Rojas M, Brodie EL, Keller K, Huber T, Dalevi D, Hu P, and Andersen GL. 2006. Greengenes, a chimera-checked 16S rRNA gene database and workbench compatible with ARB. Appl Environ Microbiol 72:5069-5072. 10.1128/AEM.03006-05

Ducarmon QR, Zwittink RD, Hornung BVH, van Schaik W, Young VB, and Kuijper EJ. 2019. Gut Microbiota and Colonization Resistance against Bacterial Enteric Infection. Microbiol Mol Biol Rev 83. 10.1128/MMBR.00007-19

Dulski T, Zakes Z, and Ciesielski S. 2018. Characterization of the gut microbiota in early life stages of pikeperch Sander lucioperca. J Fish Biol 92:94-104. 10.1111/jfb.13496

Flint HJ, Bayer EA, Rincon MT, Lamed R, and White BA. 2008. Polysaccharide utilization by gut bacteria: potential for new insights from genomic analysis. Nat Rev Microbiol 6:121-131. 10.1038/nrmicro1817

Gioia-Di Chiacchio R, Penido Junior GN, De Souza CA, Prioste FE, Prado MS, Knobl T, Menao MC, and Matushima ER. 2014. Enterobacterial colonization in captive red-eared sliders (Trachemys scripta elegans). J Zoo Wildl Med 45:919-921. 10.1638/2013-0188.1

Han X, Yang Y, Yan H, Wang X, Qu L, and Chen Y. 2015. Rumen bacterial diversity of 80 to 110-day-old goats using 16S rRNA sequencing. PLoS One 10:e0117811. 10.1371/journal.pone.0117811

Hird SM, Sanchez C, Carstens BC, and Brumfield RT. 2015. Comparative Gut Microbiota of 59 Neotropical Bird Species. Front Microbiol 6:1403. 10.3389/fmicb.2015.01403

Huang ZB, Li XY, Wang LP, and Shao ZZ. 2014. Changes in the intestinal bacterial community during the growth of white shrimp, Litopenaeus vannamei. Aquaculture Research 47:1-10.

James PG, Dittmar H, Francis LR, and F. MRJ. 2008. Detection of Salmonellae in Different Turtle 
431

432

433

434

435

436

437

438

439

440

441

442

443

444

445

446

447

448

449

450

451

452

453

454

455

456

457

458

459

460

461

462

463

464

465

466

467

468

469

470

471

Species within a Headwater Spring Ecosystem. Journal of Wildlife Diseases 44:519-526. Jandhyala SM, Talukdar R, Subramanyam C, Vuyyuru H, Sasikala M, and Nageshwar Reddy D. 2015. Role of the normal gut microbiota. World J Gastroenterol 21:8787-8803. 10.3748/wjg.v21.i29.8787

Kahrstrom CT, Pariente N, and Weiss U. 2016. Intestinal microbiota in health and disease. Nature 535:47. 10.1038/535047a

Kumar S, Indugu N, Vecchiarelli B, and Pitta DW. 2015. Associative patterns among anaerobic fungi, methanogenic archaea, and bacterial communities in response to changes in diet and age in the rumen of dairy cows. Front Microbiol 6:781. 10.3389/fmicb.2015.00781

Ley RE, Lozupone CA, Hamady M, Knight R, and Gordon JI. 2008. Worlds within worlds: evolution of the vertebrate gut microbiota. Nat Rev Microbiol 6:776-788. 10.1038/nrmicro1978

Ley RE, Turnbaugh PJ, Klein S, and Gordon JI. 2006. Microbial ecology: human gut microbes associated with obesity. Nature 444:1022-1023. 10.1038/4441022a

Li M, Zhou H, Pan X, Xu T, Zhang Z, Zi X, and Jiang Y. 2017. Cassava foliage affects the microbial diversity of Chinese indigenous geese caecum using 16S rRNA sequencing. Sci Rep 7:45697. 10.1038/srep45697

Lozupone C, and Knight R. 2005. UniFrac: a new phylogenetic method for comparing microbial communities. Appl Environ Microbiol 71:8228-8235. 10.1128/AEM.71.12.82288235.2005

Ma K, Li C, Shi HT, Wang J, Liu D, and Wan JC. 2013. Home Range Comparison of Exotic Species Trachemys scripta elegans and Native Species Mauremys sinensis in the Qionghai Section of Wanquan River, Hainan Island, China. Chinese Journal of Zoology 48:331-337.

Mali I, Wang HH, Grant WE, Feldman M, and Forstner MR. 2015. Modeling Commercial Freshwater Turtle Production on US Farms for Pet and Meat Markets. PLoS One 10:e0139053. 10.1371/journal.pone.0139053

Mariat D, Firmesse O, Levenez F, Guimaraes V, Sokol H, Dore J, Corthier G, and Furet JP. 2009. The Firmicutes/Bacteroidetes ratio of the human microbiota changes with age. BMC Microbiol 9:123. 10.1186/1471-2180-9-123

Mazmanian SK. 2008. Capsular polysaccharides of symbiotic bacteria modulate immune responses during experimental colitis. J Pediatr Gastroenterol Nutr 46 Suppl 1:E11-12. 10.1097/01.mpg.0000313824.70971.a7

Mazmanian SK, Round JL, and Kasper DL. 2008. A microbial symbiosis factor prevents intestinal inflammatory disease. Nature 453:620-625. 10.1038/nature07008

McLaughlin RW, Cochran PA, and Dowd SE. 2015. Metagenomic analysis of the gut microbiota of the Timber Rattlesnake, Crotalus horridus. Mol Biol Rep 42:1187-1195. 10.1007/s11033-015-3854-1

Miyake S, Ngugi DK, and Stingl U. 2016. Phylogenetic Diversity, Distribution, and Cophylogeny of Giant Bacteria (Epulopiscium) with their Surgeonfish Hosts in the Red Sea. Front Microbiol 7:285. 10.3389/fmicb.2016.00285

Mohd Shaufi MA, Sieo CC, Chong CW, Gan HM, and Ho YW. 2015. Deciphering chicken gut 
microbial dynamics based on high-throughput $16 \mathrm{~S}$ rRNA metagenomics analyses. Gut Pathog 7:4. 10.1186/s13099-015-0051-7

Nishizawa H, Tabata R, Hori T, Mitamura H, and Arai N. 2014. Feeding kinematics of freshwater turtles: what advantage do invasive species possess? Zoology (Jena) 117:315-318. 10.1016/j.zool.2014.04.005

Polo-Cavia N, Lopez P, and Martin J. 2012. Feeding status and basking requirements of freshwater turtles in an invasion context. Physiol Behav 105:1208-1213. 10.1016/j.physbeh.2011.12.020

Price JT, Paladino FV, Lamont MM, Witherington BE, Bates ST, and Soule T. 2017. Characterization of the juvenile green turtle (Chelonia mydas) microbiome throughout an ontogenetic shift from pelagic to neritic habitats. PLoS One 12:e0177642. 10.1371/journal.pone.0177642

Qin J, Li R, Raes J, Arumugam M, Burgdorf KS, Manichanh C, Nielsen T, Pons N, Levenez F, Yamada T, Mende DR, Li J, Xu J, Li S, Li D, Cao J, Wang B, Liang H, Zheng H, Xie Y, Tap J, Lepage P, Bertalan M, Batto JM, Hansen T, Le Paslier D, Linneberg A, Nielsen HB, Pelletier E, Renault P, Sicheritz-Ponten T, Turner K, Zhu H, Yu C, Li S, Jian M, Zhou Y, Li Y, Zhang X, Li S, Qin N, Yang H, Wang J, Brunak S, Dore J, Guarner F, Kristiansen K, Pedersen O, Parkhill J, Weissenbach J, Meta HITC, Bork P, Ehrlich SD, and Wang J. 2010. A human gut microbial gene catalogue established by metagenomic sequencing. Nature 464:59-65. 10.1038/nature08821

Quast C, Pruesse E, Yilmaz P, Gerken J, Schweer T, Yarza P, Peplies J, and Glockner FO. 2013. The SILVA ribosomal RNA gene database project: improved data processing and webbased tools. Nucleic Acids Res 41:D590-596. 10.1093/nar/gks1219

Ravussin Y, Koren O, Spor A, LeDuc C, Gutman R, Stombaugh J, Knight R, Ley RE, and Leibel RL. 2012. Responses of gut microbiota to diet composition and weight loss in lean and obese mice. Obesity (Silver Spring) 20:738-747. 10.1038/oby.2011.111

Rawls JF, Samuel BS, and Gordon JI. 2004. Gnotobiotic zebrafish reveal evolutionarily conserved responses to the gut microbiota. Proc Natl Acad Sci U S A 101:4596-4601. 10.1073/pnas.0400706101

Sarasquete MC, Polo A, and YúFera M. 1995. Histology and histochemistry of the development of the digestive system of larval gilthead seabream, Sparus aurata L. Aquaculture 130:7992.

Scott KP, Antoine JM, Midtvedt T, and van Hemert S. 2015. Manipulating the gut microbiota to maintain health and treat disease. Microb Ecol Health Dis 26:25877. 10.3402/mehd.v26.25877

Scott KP, Gratz SW, Sheridan PO, Flint HJ, and Duncan SH. 2013. The influence of diet on the gut microbiota. Pharmacol Res 69:52-60. 10.1016/j.phrs.2012.10.020

Segata N, Izard J, Waldron L, Gevers D, Miropolsky L, Garrett WS, and Huttenhower C. 2011. Metagenomic biomarker discovery and explanation. Genome Biol 12:R60. 10.1186/gb2011-12-6-r60

Sergeant MJ, Constantinidou C, Cogan TA, Bedford MR, Penn CW, and Pallen MJ. 2014. 
513

514

515

516

517

518

519

520

521

522

523

524

525

526

527

528

529

530

531

532

533

534

535

536

537

538

539

540

541

542

543

544

545

Extensive microbial and functional diversity within the chicken cecal microbiome. PLoS One 9:e91941. 10.1371/journal.pone.0091941

Simpson EH. 1949. Measurement of diversity. Nature 163:688-688.

Singh KM, Shah T, Deshpande S, Jakhesara SJ, Koringa PG, Rank DN, and Joshi CG. 2012. High through put 16S rRNA gene-based pyrosequencing analysis of the fecal microbiota of high FCR and low FCR broiler growers. Mol Biol Rep 39:10595-10602. 10.1007/s11033-0121947-7

Sun DL, Jiang X, Wu QL, and Zhou NY. 2013. Intragenomic heterogeneity of 16S rRNA genes causes overestimation of prokaryotic diversity. Appl Environ Microbiol 79:5962-5969. 10.1128/AEM.01282-13

Thomas F, Hehemann JH, Rebuffet E, Czjzek M, and Michel G. 2011. Environmental and gut bacteroidetes: the food connection. Front Microbiol 2:93. 10.3389/fmicb.2011.00093

Wang Q, Garrity GM, Tiedje JM, and Cole JR. 2007. Naive Bayesian classifier for rapid assignment of rRNA sequences into the new bacterial taxonomy. Appl Environ Microbiol 73:5261-5267. 10.1128/AEM.00062-07

Yamashiro Y. 2017. Gut Microbiota in Health and Disease. Ann Nutr Metab 71:242-246. $10.1159 / 000481627$

Yang JB, and Shi HT. 2014. Home Range of Trachemys scripta elegans in Brackish Water of Nandu River, Hainan Island, China. Sichuan Journal of Zoology 33:685-688.

Yu C, Q., Han F, S., and Fu G. 2019. Effects of 7 years experimental warming on soil bacterial and fungal community structure in the Northern Tibet alpine meadow at three elevations. Science of the Total Environment 655:814-822.

Zeng B, Han S, Wang P, Wen B, Jian W, Guo W, Yu Z, Du D, Fu X, Kong F, Yang M, Si X, Zhao $\mathrm{J}$, and Li Y. 2015. The bacterial communities associated with fecal types and body weight of rex rabbits. Sci Rep 5:9342. 10.1038/srep09342

Zha Y, Eiler A, Johansson F, and Svanback R. 2018. Effects of predation stress and food ration on perch gut microbiota. Microbiome 6:28. 10.1186/s40168-018-0400-0

Zhao LH, Li J, Wei CJ, Wang JC, Hone ML, and Shi HT. 2013. Comparisons on the Competitive Ability for Food between Trachemys scripta elegans and Mauremys sinensis. Sichuan Journal of Zoology 32:873-878.

Zhao W, Wang Y, Liu S, Huang J, Zhai Z, He C, Ding J, Wang J, Wang H, Fan W, Zhao J, and Meng H. 2015. The dynamic distribution of porcine microbiota across different ages and gastrointestinal tract segments. PLoS One 10:e0117441. 10.1371/journal.pone.0117441. 


\section{Table $\mathbf{1}$ (on next page)}

Table 1. Physiological characterization of red-eared slider turtles at different growth stages

${ }^{x}$ Values sharing the same superscript letters are not significantly different from each other as determined by a multiple comparison test of least significant difference (LSD) $(p<0.05)$. 
1

\begin{tabular}{lllll}
\hline Physiological index & 0 day & 10 day & 20 day & 30 day \\
\hline Body weight $(\mathrm{g})$ & $8.25 \pm 0.55^{\mathrm{ax}}$ & $7.53 \pm 0.58^{\mathrm{a}}$ & $9.31 \pm 0.90^{\mathrm{b}}$ & $10.59 \pm 1.19^{\mathrm{c}}$ \\
Carapace length $(\mathrm{mm})$ & $32.20 \pm 1.07^{\mathrm{a}}$ & $32.20 \pm 1.15^{\mathrm{a}}$ & $34.51 \pm 0.81^{\mathrm{b}}$ & $37.56 \pm 0.83^{\mathrm{c}}$ \\
Carapace width $(\mathrm{mm})$ & $30.73 \pm 1.09^{\mathrm{a}}$ & $31.30 \pm 0.96^{\mathrm{a}}$ & $33.60 \pm 0.75^{\mathrm{b}}$ & $36.57 \pm 0.98^{\mathrm{c}}$ \\
Body height $(\mathrm{mm})$ & $16.38 \pm 0.58^{\mathrm{ac}}$ & $15.12 \pm 0.58^{\mathrm{b}}$ & $15.88 \pm 0.93^{\mathrm{ab}}$ & $17.04 \pm 0.78^{\mathrm{c}}$ \\
\hline
\end{tabular}




\section{Table 2 (on next page)}

Table 2. Bacterial richness and diversity in intestine in red-eared sliders at different growth stages and water

Reads indicate the number of sequences archived by high-throughput sequencing. OTUs were defined at $3 \%$ dissimilarity. The richness estimators (ACE and Chao) and diversity indices (Shannon and Simpson) were calculated.

Date are expressed as adjusted Mean $\pm \mathrm{SE}$, with body weight as the covariate. Covariates appearing in the model are evaluated at the following values: body weight $=8.92 \mathrm{~g}$

${ }^{x}$ Values sharing the same superscript letters are not significantly different from each other according to a multiple comparison test of least significant difference (LSD) $(p<0.05)$. 
1

\begin{tabular}{llllllll}
\hline & Group & Reads & OTUs & Chao1 & Ace & Shannon & Simpson \\
\hline Intestine & 0 day & $53419.0 \pm 5781.6$ & $751.7 \pm 285.3^{\mathrm{x}}$ & $2196.6 \pm 281.3^{\mathrm{a}}$ & $2186.5 \pm 304.0^{\mathrm{a}}$ & $2.50 \pm 0.81^{\mathrm{a}}$ & $0.63 \pm 0.16^{\mathrm{a}}$ \\
& 10 day & $41455.0 \pm 12238.2$ & $1532.7 \pm 337.0^{\mathrm{b}}$ & $4974.1 \pm 1118.8^{\mathrm{b}}$ & $5224.1 \pm 1118.0^{\mathrm{b}}$ & $4.41 \pm 0.36^{\mathrm{b}}$ & $0.88 \pm 0.031^{\mathrm{b}}$ \\
& 20 day & $52621.7 \pm 5297.6$ & $1511.3 \pm 99.0^{\mathrm{b}}$ & $4935.0 \pm 356.8^{\mathrm{b}}$ & $5190.6 \pm 557.1^{\mathrm{b}}$ & $3.48 \pm 0.37^{\mathrm{ab}}$ & $0.74 \pm 0.58^{\mathrm{a}}$ \\
& 30 day & $45357.3 \pm 5474.2$ & $1262.0 \pm 88.5^{\mathrm{ab}}$ & $3475.8 \pm 546.7^{\mathrm{a}}$ & $3444.4 \pm 558.6^{\mathrm{a}}$ & $4.35 \pm 0.15^{\mathrm{b}}$ & $0.88 \pm 0.0058^{\mathrm{b}}$ \\
Environment & Water & $44824.7 \pm 9255.9$ & $680.7 \pm 167.4$ & $1533.2 \pm 369.4$ & $1465.5 \pm 482.5$ & $4.36 \pm 0.74$ & $0.87 \pm 0.053$ \\
\hline
\end{tabular}

2 
Figure 1

Figure 1. Beta diversity analysis of microbiomes from turtle intestines at different growth stages and of the environmental water ( $n=3$ samples of each condition).

Contribution of different taxonomic groups to separation of samples based on phylogenetic information. The samples were clustered by PCoA plots using weighted UniFrac distances.

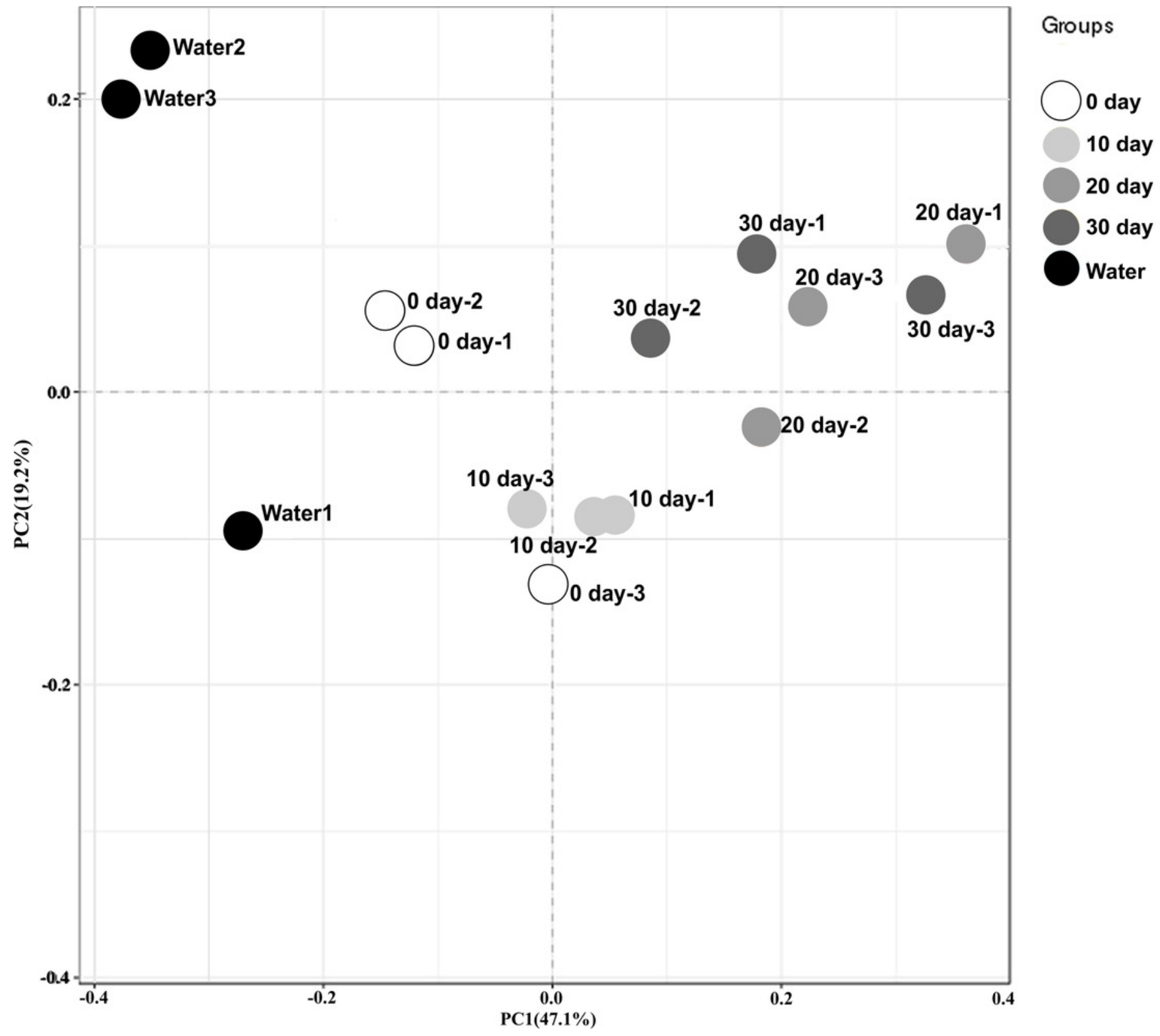




\section{Figure 2}

Figure 2. Taxonomic compositions of turtle intestinal microbiomes and water microbiomes from different samples at the phylum level.

(A) Heat map of the microbial composition of the red-eared slider gastrointestinal tract, and the water with row normalized. The heat map indicates the relative abundance of each phylum in different samples. (B) Relative abundance of sequences belonging to the dominant phyla from different samples. A color and shadow-coded bar plot shows the average bacterial phylum distribution in different samples. Sequences that could not be classified into any known group were assigned as 'Others'. 
A

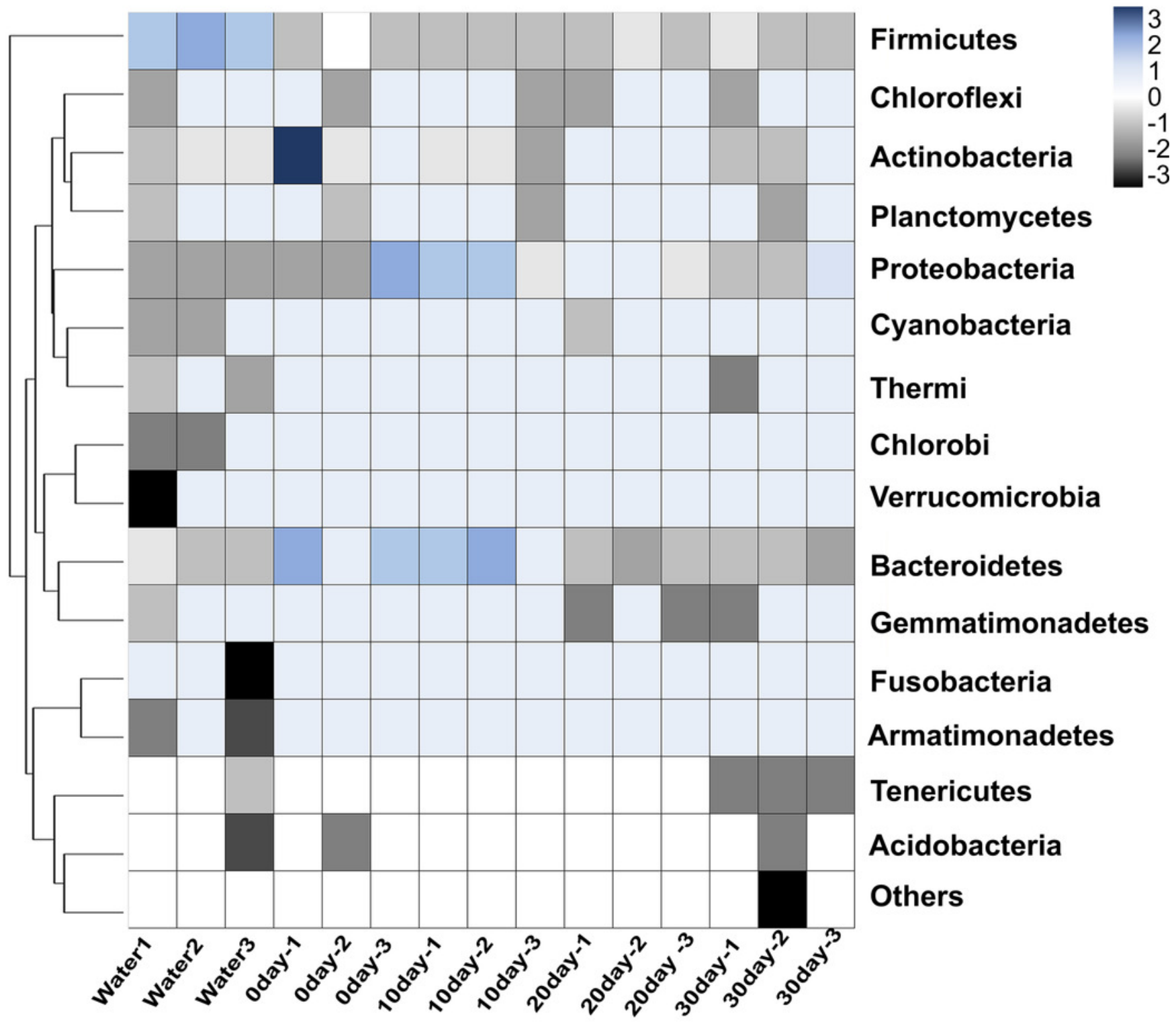

B

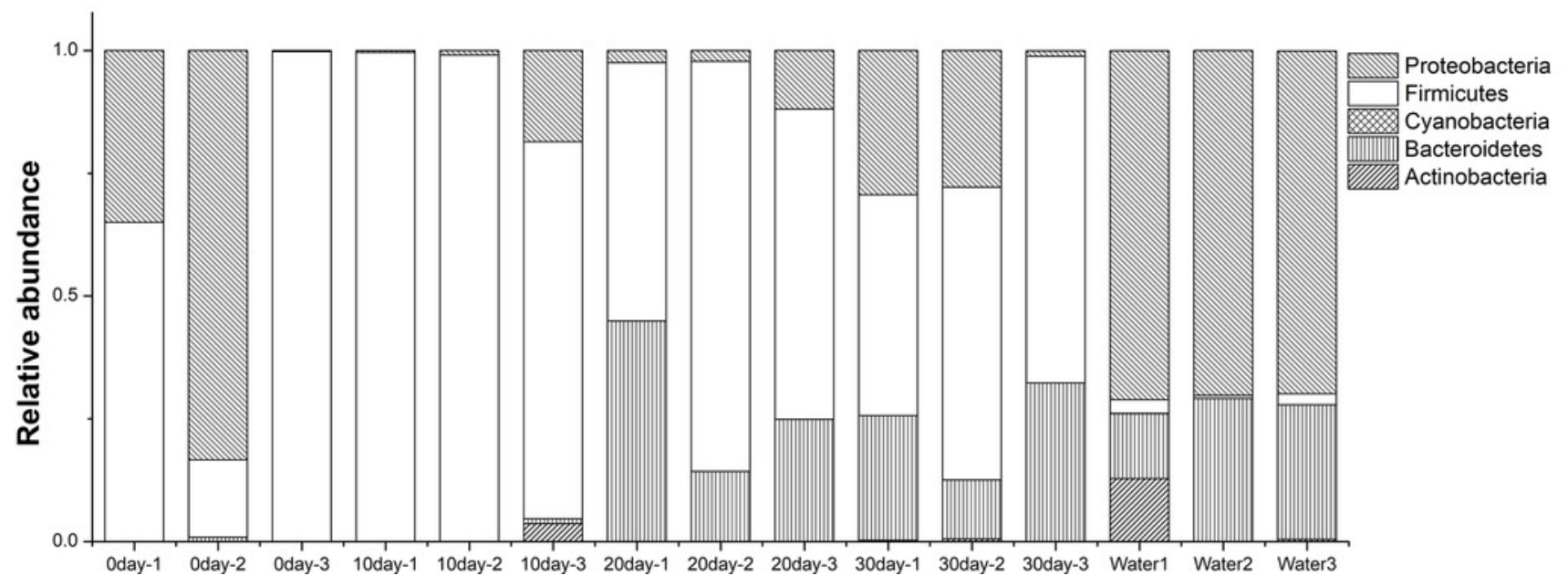


Figure 3

Figure 3. Relative composition of the intestinal microbiome of red-eared sliders for different growth time (A) and the water microbiome (B) at the family level.

A color and shadow-coded bar plot shows the average bacterial family distribution in different samples

A

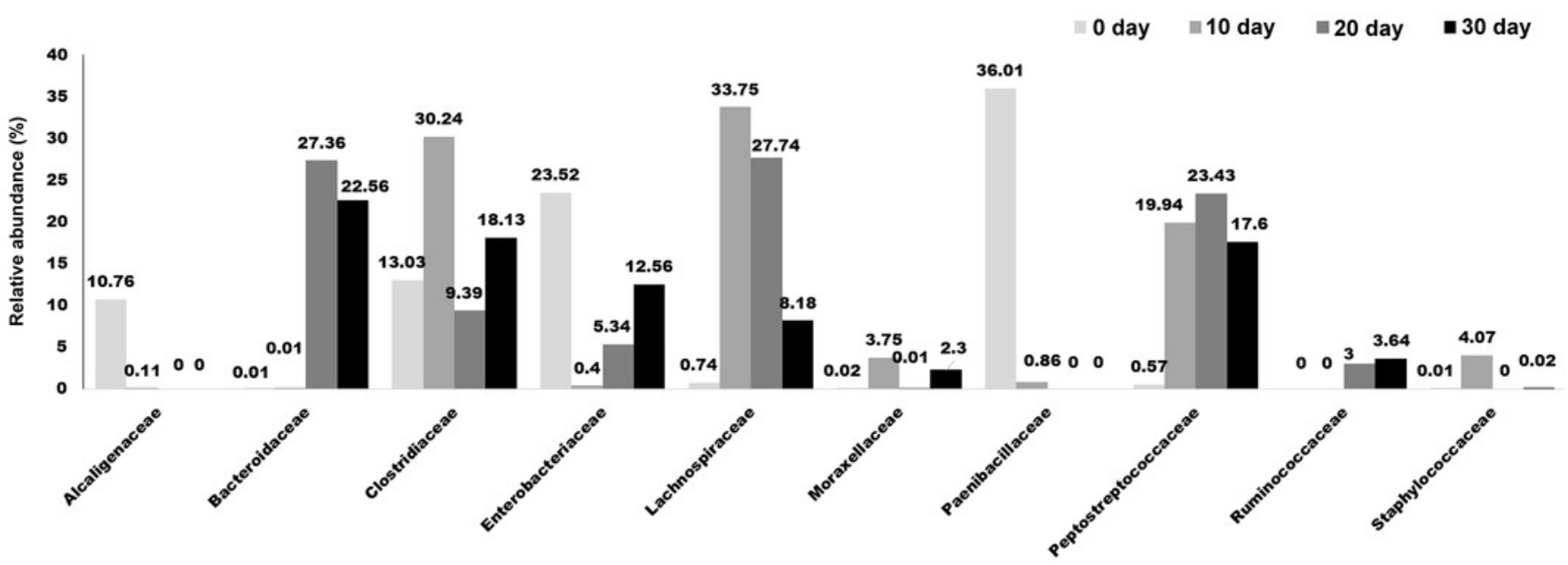

B

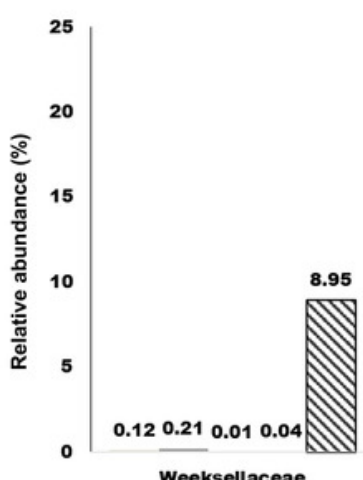

Weeksellaceae

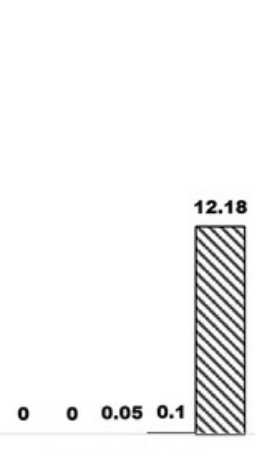

Aeromonadaceae

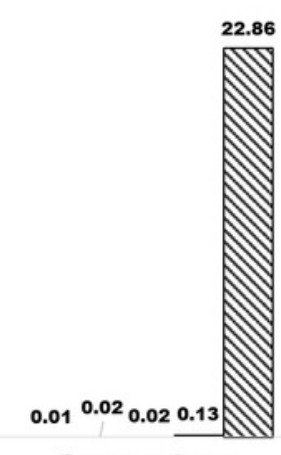

Comamonadaceae
0 day 10 day $\mathbf{2 0}$ day $\square \mathbf{3 0}$ day $\triangle W$ Water

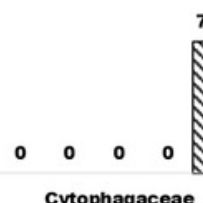

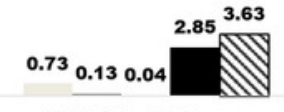

Methylobacteriaceae
$0.37^{1.27} 0.010 .03$

Xanthomonadaceae 
Figure 4

Figure 4. Analysis of the differences in the intestinal microbiome among different growth stages in turtles.

Venn diagrams showing the number of shared and exclusive OTUs in different growth stage turtles. 


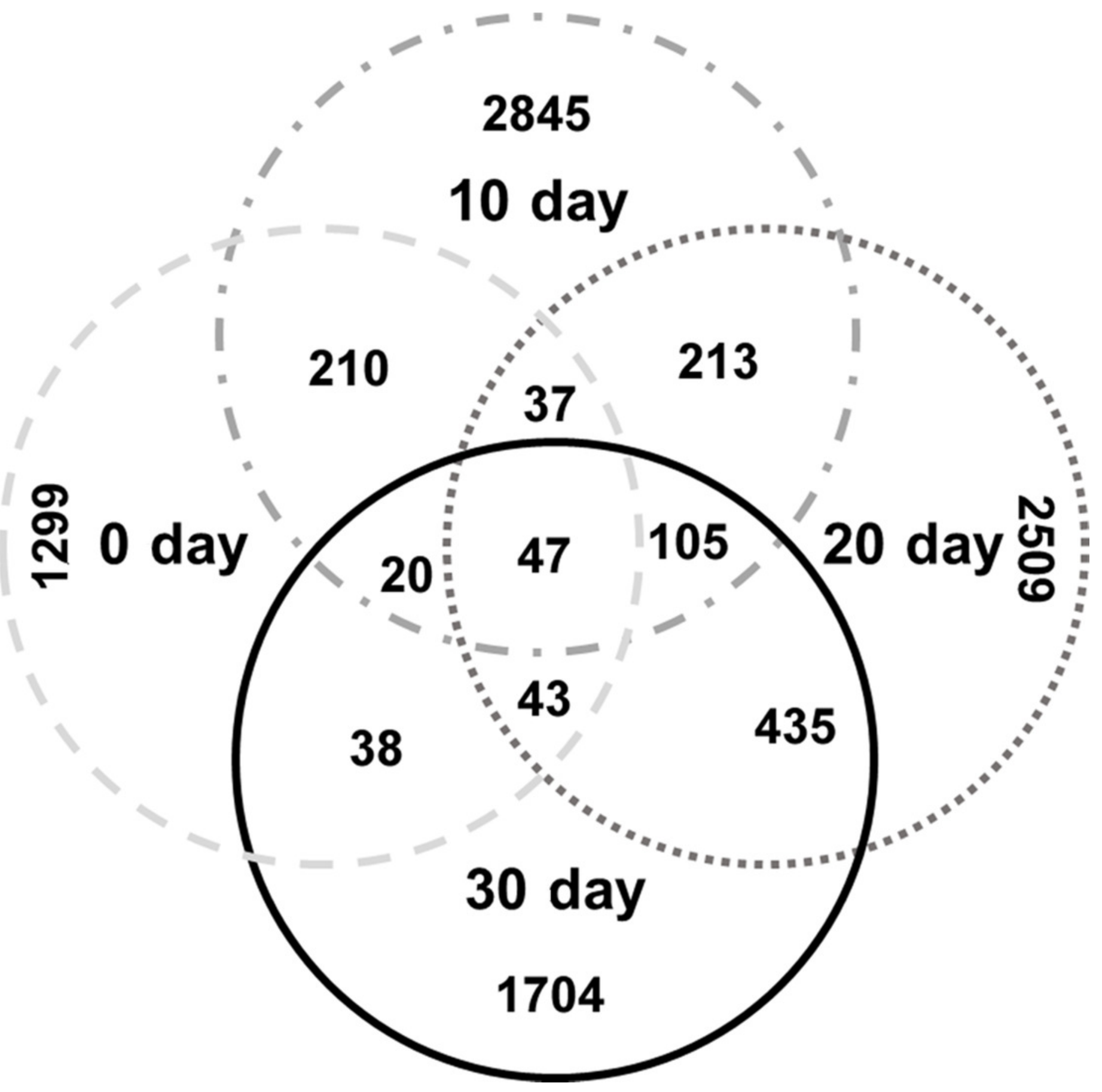




\section{Figure 5}

Figure 5. LEfSe identified the most differentially abundant bacterial taxa at different growth stages of turtles at different taxonomic levels.

0 day, 10 day, 20 day, 30 day, indicating the intestinal microbiome of turtles growing 0 day, 10days, 20 days, 30 days. Only taxa meeting an LDA significant threshold $>2$ are shown. The taxa could be regarded as a biomarker when LDA significant threshold $>4$, and it was marked by the sign of "star". (The eggs and turtles drawings in the figure are original and drawn by Qin Peng). 
Family:Porphyromonadaceae Family:Peptococcaceae Family:Veillonellaceae Family:Erysipelotrichaceae Family:Cytophagaceae Genus:Clavibacte Genus:Chelativorans Genus:Blautia

Genus:Anaerotruncus Genus:Ruminococcus Genus:Holdemania Species:producta Species:gnavus

Family:Bacteroidaceae Family:Odoribacteraceae Family:Turicibacteraceae Genus:Coprococcus Genus:Dorea Genus:Eubacterium Genus:Faecalibacterium Genus:Odoribacter Genus:Turicibacte Species:butyricum Species:dolichum

Family:Brevibacteriaceae Family:Lachnospiraceae Family:Planococcaceae Genus:Brevibacterium Genus:Epulopiscium Genus:Janibacte Genus:Kurthia Species:aureum Species:gibsonii

Family:Alcaligenaceae Family:Paenibacillaceae Family:Sphingobacteriaceae

Genus:Achromobacter Genus:Bacillus

Genus:Ochrobactrum

Genus:Paenibacillus Genus:Sphingobacterium Species:firmus Species:lautus
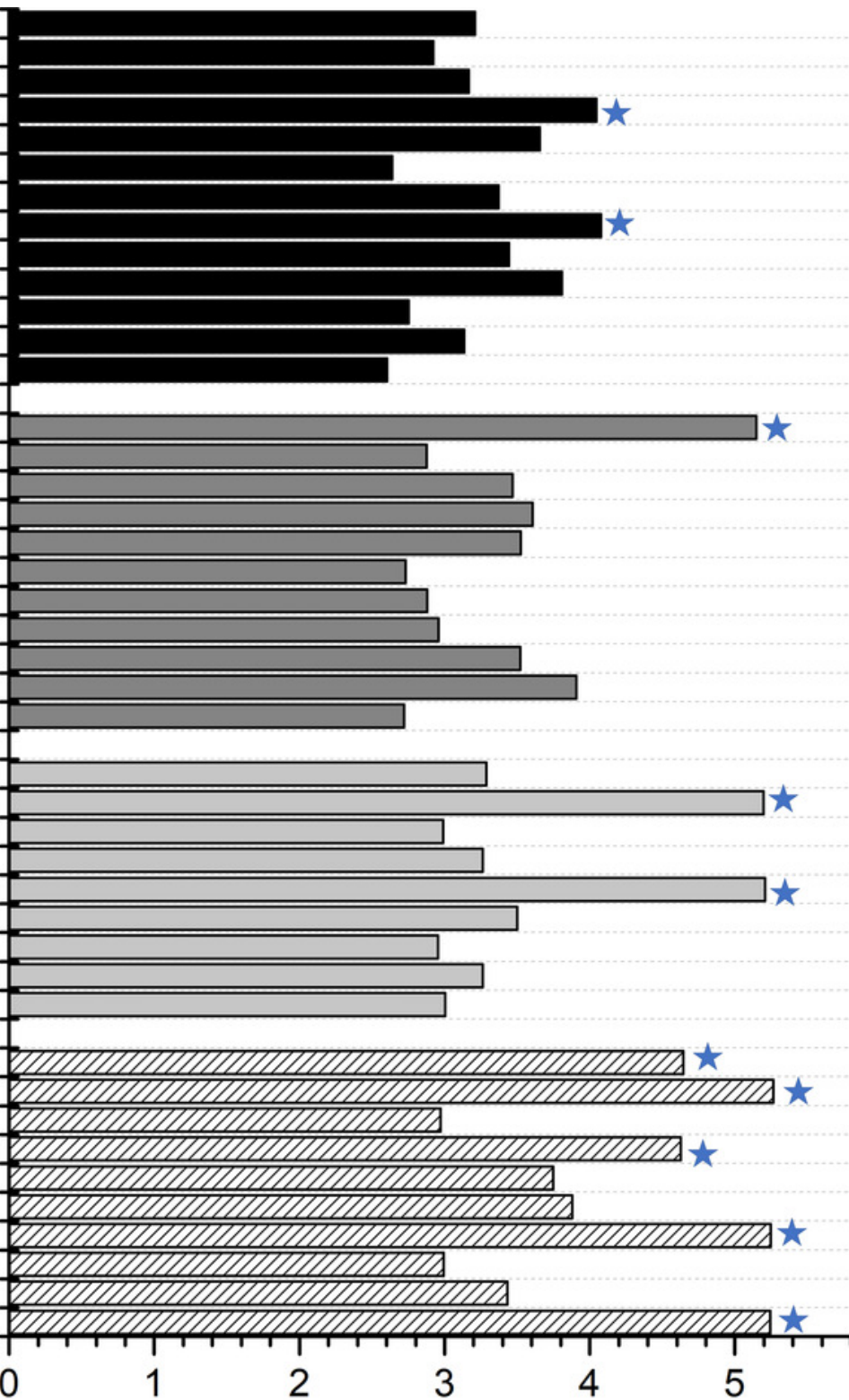

0

LDA Score $\left(\log _{10}\right)$
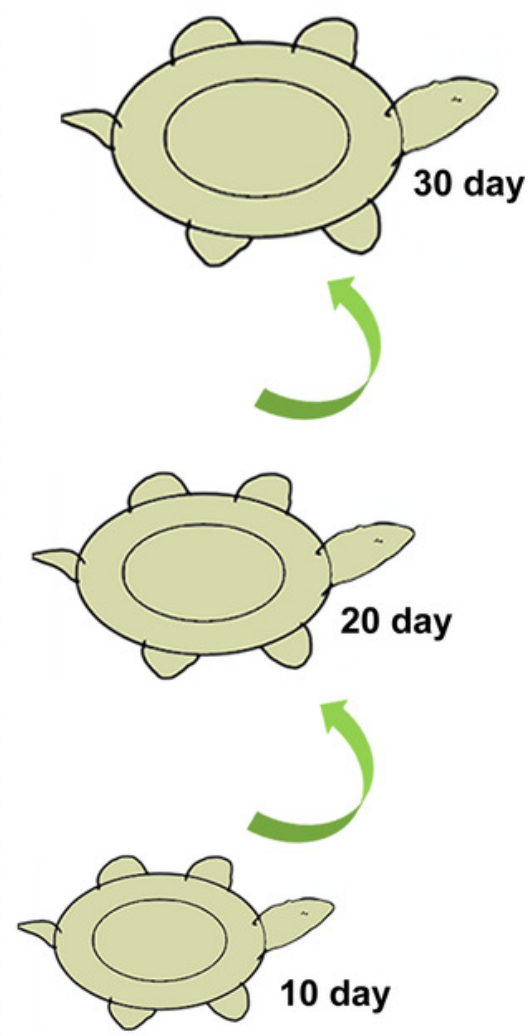

$\mathbf{0}$ day 